\title{
Larvicidal Efficacy of Jasminum sp. (Oleaceae) Flower Extracts against the Dengue and Chikungunya Vector Aedes aegypti L. (Diptera: Culicidae)
}

\section{Eugeni Anitha Preethi $\mathbf{G}^{1}$, Raveen $\mathbf{R}^{1 *}$, Arivoli $\mathbf{S}^{2}$, Samuel Tennyson ${ }^{1}$ and Madhanagopal $\mathbf{R}^{3}$}

${ }^{1}$ Department of Zoology, Madras Christian College, Chennai 600 059, Tamil Nadu, India

${ }^{2}$ Department of Zoology, Thiruvalluvar University, Vellore 632 115, Tamil Nadu, India

${ }^{3}$ Department of Statistics, Madras Christian College, Chennai 600 059, Tamil Nadu, India

\begin{abstract}
Dengue and chikungunya are transmitted by Aedes aegypti and for controlling these diseases, the vector mosquito has to be controlled. Extensive use of synthetic and chemical insecticides has resulted in environmental hazards and also in development of physiological resistance among vector mosquito species. Plant products are considered to be a potential alternative approach as they are environmentally safe, target specific and biodegradable. In the present study, the crude chloroform, methanol and aqueous flower extracts of Jasminum officinale, Jasminum auriculatum and Jasminum grandiflorum were tested for the larvicidal efficacy against the third instar larvae of Aedes aegypti at concentrations of $62.5,125,250,500,1000,2000,4000$ and $8000 \mathrm{mg} / \mathrm{L}$. Mortality was recorded after 24 and $48 \mathrm{~h}$. Amongst the extracts of Jasminum species tested, the crude chloroform flower extract of Jasminum grandiflorum was found to be effective showing $100 \%$ mortality at $1000 \mathrm{mg} / \mathrm{L}$ with $\mathrm{LC}_{50}$ value of 344.01 and 300.47 after 24 and $48 \mathrm{~h}$ respectively followed by the crude methanolic flower extracts of Jasminum officinale and Jasminum auriculatum. Further investigations are needed to elucidate the larvicidal activity of Jasminum grandiflorum crude chloroform flower extract against a wide range of all stages of mosquito species and also the active ingredient(s) of the extract responsible for larvicidal activity should be identified.
\end{abstract}

Keywords: Larvicidal efficacy; Aedes aegypti; Jasminum officinale; Jasminum auriculatum; Jasminum grandiflorum; Crude flower extracts

\section{Introduction}

Human beings are being suffered from the menace of mosquitoes since time immemorial and it is believed that mosquitoes are ranked as the most important pests causing human health concerns, since they are responsible for transmission of dengue, dengue haemorrhagic fever, chikungunya, malaria, filarial fever and Japanese encephalitis that cause severe public health problems [1]. Vector control is an important component in a disease control programmme. A myriad of methods and strategies though available, may not contribute to the total control of vectors unless they are used judiciously and in a sustained manner. The discovery and development of synthetic organic chemicals with persistent residual action not only over shadow the use of herbal products against mosquitoes, but also become the major weapon for mosquito control. But the extensive use of synthetic organic insecticides has resulted in environmental hazards, ecological imbalance, harm to humans and animals and non target organisms being affected, in addition to the physiological resistance of vectors [2]. This has necessitated the need for search and development of environmental-safe, biodegradable and indigenous method for vector control. The flora of India has a rich aromatic plant diversity with potential for development of natural insecticides for the control of mosquito and other pests [3]. Phytochemical insecticides have received much attention, in this regard, as they are considered to be more environmentally biodegradable and considered safer than synthetic insecticides [4]. Therefore, the search for such compounds has been directed extensively to the plant kingdom. Co-evolution has equipped plants with a plethora of chemical defenses against insects. Aware of this effect, mankind has used plant parts or extracts to control insects/mosquitoes since ancient time. Plant derived products have received increased attention from scientists as they are a rich source of novel natural substances possessing insecticidal properties, safer to humans and ecosystem [5]. During the last decade, various studies on natural plant products against vector mosquito indicate them as possible alternatives to chemical synthetic insecticides for mosquito control [6-13]. Therefore, in the present study, the crude flower extracts of Jasminum officinale, Jasminum auriculatum and Jasminum grandiflorum were tested for the larvicidal efficacy against the third instar larvae of Aedes aegypti.

\section{Materials and Methods}

\section{Plant collection and extraction}

Mature fresh flowers of Jasminum officinale, Jasminum auriculatum and Jasminum grandiflorum collected in and around Chennai, Tamil Nadu, India were brought to the laboratory, shade dried at room temperature and powdered. Dried and powdered flowers $(1 \mathrm{~kg})$ each was macerated with $3 \mathrm{~L}$ of chloroform, methanol and distilled water for a period of $96 \mathrm{~h}$ each separately and filtered. The filtrate was then concentrated at reduced temperature on a rotary evaporator. The crude chloroform, methanol and aqueous flower extracts of Jasminum officinale, Jasminum auriculatum and Jasminum grandiflorum thus obtained were lyophilized and a stock solution of 1,00,000 mg/L prepared by adding adequate volume of acetone was refrigerated at $4^{\circ} \mathrm{C}$ until testing for bioassay.

\section{Test mosquitoes}

Aedes immatures collected from various places in Chennai, Tamil $\mathrm{Nadu}$, India were transported to the laboratory in plastic containers. In the laboratory, the immature mosquitoes were transferred to

*Corresponding authors: Dr. Raveen, Assistant Professor, Department of Zoology, Madras Christian College, Chennai-600 059, Tamil Nadu, India, Tel: 91 9840463064; E-mail: raveenraja2002@yahoo.co.in

Received September 05, 2014; Accepted September 26, 2014; Published September 28, 2014

Citation: Eugeni Anitha Preethi G, Raveen R, Arivoli S, Samuel T, Madhanagopa $\mathrm{R}$ (2014) Larvicidal Efficacy of Jasminum sp. (Oleaceae) Flower Extracts agains the Dengue and Chikungunya Vector Aedes aegypti L. (Diptera: Culicidae). Med chem 4: 672-675. doi:10.4172/2161-0444.1000210

Copyright: (c) 2014 Eugeni Anitha Preethi G, et al. This is an open-access article distributed under the terms of the Creative Commons Attribution License, which permits unrestricted use, distribution, and reproduction in any medium, provided the original author and source are credited. 
Citation: Eugeni Anitha Preethi G, Raveen R, Arivoli S, Samuel T, Madhanagopal R (2014) Larvicidal Efficacy of Jasminum sp. (Oleaceae) Flower Extracts against the Dengue and Chikungunya Vector Aedes aegypti L. (Diptera: Culicidae). Med chem 4: 672-675. doi:10.4172/21610444.1000210

enamel larval trays until adult emergence. After emergence, the adult mosquitoes were identified upto species level and confirmed before rearing. Cyclic generations of Aedes aegypti were maintained separately in two feet mosquito cages in an insectary. Mean room temperature of $27 \pm 2^{\circ} \mathrm{C}$ and a relative humidity of $70-80 \%$ were maintained in the insectary. The adult mosquitoes were fed on ten per cent glucose solution. For continuous maintenance of mosquito colony, the adult female mosquitoes were blood fed with laboratory reared albino mice. Ovitraps were placed inside the cages for egg laying. The eggs laid were then transferred to enamel larval trays maintained in the larval rearing chamber. The larvae were fed with larval food (dog biscuits and yeast in the ratio $3: 1$ ). The larvae on becoming pupae were collected, transferred to plastic bowls and kept inside mosquito cage for adult emergence.

\section{Larvicidal bioassay}

Standard WHO [14] protocol with minor modifications was adopted for the study. The tests were conducted in glass beakers. Aedes aegypti immature particularly early third instar larvae were obtained from laboratory colonized mosquitoes of $\mathrm{F}_{1}$ generation. From the stock solutions, concentrations of $62.5,125,250,500,1000$, 2000, 4000 and $8000 \mathrm{mg} / \mathrm{L}$ were prepared. Twenty healthy larvae were released into each $250 \mathrm{ml}$ glass beaker containing $200 \mathrm{~mL}$ of water and test concentration. Mortality was observed for 24 and $48 \mathrm{~h}$ after treatment. A total of three trials with three replicates per trial for each concentration were carried out. Controls were run simultaneously. Treated control was prepared by the addition of acetone to distilled water. Distilled water served as untreated control. The larval per cent mortality was calculated and when control mortality ranged from 5-20\% it was corrected using Abbott's formula [15]. SPSS 11.5 version package was used for determination of $\mathrm{LC}_{50}$ and $\mathrm{LC}_{90}$ values [16]. One way ANOVA followed by Tukey's test was performed to determine the difference in larval mortality between concentrations.

\section{Results}

Results of the larvicidal effects of crude flower extracts of Jasminum species against Aedes aegypti are presented in Tables 1 and 2. Among the plant species and extracts tested, the crude chloroform flower of Jasminum grandiflorum was found to be effective followed by the crude methanol flower extracts of Jasminum officinale and Jasminum auriculatum. One hundred per cent mortality was observed in Jasminum grandiflorum crude chloroform flower extract at $1000 \mathrm{mg} / \mathrm{L}$ at 24 hours (Tables 3 and 4). The crude chloroform flower extract Jasminum grandiflorum was found to be effective and promising with $\mathrm{LC}_{50}$ values of 344.01 and $300.47 \mathrm{mg} / \mathrm{L}$ after 24 and $48 \mathrm{~h}$ respectively (Table 5).

\section{Discussion}

Vector control is facing a threat due to the emergence of resistance in vector mosquitoes to conventional synthetic insecticides, warranting counter measures such as developmental of novel insecticides [17]. Mosquitoes in the larval stage are attractive targets for pesticides because mosquitoes breed in water, and thus, it is easy to deal with them in this habitat. Mosquito control approaches based on synthetic insecticides have created many problems like insecticide resistance [18]. Natural products of plant origin with insecticidal properties have been tried in the recent past in order to control a variety of insect pests and vectors. This has necessitated the need for a research and development of environmentally safe, biodegradable indigenous method for vector control. Many researchers have reported on the effectiveness of plant extract against mosquito larvae [19-21]. Phytoextracts are emerging as potential mosquito control agents, and can be used successfully in mosquito management programmes [22]. The results of the present study indicate the crude chloroform extract of Jasminum grandiflorum flowers to possess larvicidal activity against Aedes aegypti.

\begin{tabular}{|c|c|c|c|c|c|c|c|c|c|c|c|}
\hline \multirow{2}{*}{ Plant species } & \multirow{2}{*}{ Solvents } & \multicolumn{10}{|c|}{ Concentration (mg/L) } \\
\hline & & UC & TC & 62.5 & 125 & 250 & 500 & 1000 & 2000 & 4000 & 8000 \\
\hline \multirow{3}{*}{$\begin{array}{l}\text { Jasminum } \\
\text { officinale }\end{array}$} & Chloroform & $0.00 \pm 0.00^{\mathrm{a}}$ & $0.00 \pm 0.00^{\mathrm{a}}$ & $0.00 \pm 0.00^{\mathrm{a}}$ & $0.00 \pm 0.00^{\mathrm{a}}$ & $0.00 \pm 0.00^{\mathrm{a}}$ & $0.00 \pm 0.00^{\mathrm{a}}$ & $0.00 \pm 0.00^{\mathrm{a}}$ & $0.00 \pm 0.00^{\mathrm{a}}$ & $0.00 \pm 0.00^{\mathrm{a}}$ & $1.00 \pm 1.00^{b}$ \\
\hline & Methanol & $0.00 \pm 0.00^{\mathrm{a}}$ & $0.00 \pm 0.00^{\mathrm{a}}$ & $0.33 \pm 0.57^{a}$ & $0.33 \pm 0.57^{\mathrm{a}}$ & $0.66 \pm 0.57^{a}$ & $0.66 \pm 0.57^{a}$ & $0.66 \pm 0.57^{\mathrm{a}}$ & $1.33 \pm 1.15^{\mathrm{ab}}$ & $3.66 \pm 1.15^{b}$ & $3.66 \pm 2.08^{b}$ \\
\hline & Aqueous & $0.00 \pm 0.00^{\mathrm{a}}$ & $0.00 \pm 0.00^{\mathrm{a}}$ & $0.00 \pm 0.00^{\mathrm{a}}$ & $0.00 \pm 0.00^{\mathrm{a}}$ & $0.00 \pm 0.00^{\mathrm{a}}$ & $0.00 \pm 0.00^{\mathrm{a}}$ & $0.00 \pm 0.00^{\mathrm{a}}$ & $0.00 \pm 0.00^{\mathrm{a}}$ & $0.33 \pm 0.57^{\mathrm{a}}$ & $0.66 \pm 1.15^{\mathrm{a}}$ \\
\hline \multirow{3}{*}{$\begin{array}{c}\text { Jasminum } \\
\text { auriculatum }\end{array}$} & Chloroform & $0.00 \pm 0.00^{\mathrm{a}}$ & $0.00 \pm 0.00^{\mathrm{a}}$ & $0.00 \pm 0.00^{\mathrm{a}}$ & $0.33 \pm 0.57^{\mathrm{ab}}$ & $0.33 \pm 0.57^{\mathrm{ab}}$ & $0.66 \pm 0.57^{\mathrm{abc}}$ & $1.33 \pm 0.57^{\mathrm{abc}}$ & $1.66 \pm 1.52^{\mathrm{abc}}$ & $2.00 \pm 0.00^{\text {bc }}$ & $2.33 \pm 0.57^{c}$ \\
\hline & Methanol & $0.00 \pm 0.00^{\mathrm{a}}$ & $0.00 \pm 0.00^{\mathrm{a}}$ & $0.00 \pm 0.00^{\mathrm{a}}$ & $0.00 \pm 0.00^{\mathrm{a}}$ & $0.00 \pm 0.00^{\mathrm{a}}$ & $0.00 \pm 0.00^{\mathrm{a}}$ & $0.00 \pm 0.00^{\mathrm{a}}$ & $1.00 \pm 1.00^{\mathrm{a}}$ & $4.00 \pm 2.64^{b}$ & $4.00 \pm 1.00^{b}$ \\
\hline & Aqueous & $0.00 \pm 0.00^{\mathrm{a}}$ & $0.00 \pm 0.00^{\mathrm{a}}$ & $0.00 \pm 0.00^{\mathrm{a}}$ & $0.66 \pm 0.57^{\mathrm{ab}}$ & $1.00 \pm 1.00^{\mathrm{ab}}$ & $1.33 \pm 0.57^{\mathrm{ab}}$ & $1.33 \pm 0.57^{\mathrm{ab}}$ & $1.66 \pm 0.57^{\mathrm{ab}}$ & $2.00 \pm 1.00^{\mathrm{b}}$ & $2.00 \pm 0.00^{\mathrm{b}}$ \\
\hline \multirow{3}{*}{$\begin{array}{c}\text { Jasminum } \\
\text { grandiflorum }\end{array}$} & Chloroform & $0.00 \pm 0.00^{\mathrm{a}}$ & $0.00 \pm 0.00^{\mathrm{a}}$ & $6.66 \pm 0.57^{b}$ & $7.00 \pm 1.00^{b}$ & $7.33 \pm 2.08^{b}$ & $10.66 \pm 2.08^{c}$ & $20.00 \pm 0.00^{d}$ & $20.00 \pm 0.00^{d}$ & $20.00 \pm 0.00^{d}$ & $20.00 \pm 0.00^{d}$ \\
\hline & Methanol & $0.00 \pm 0.00^{\mathrm{a}}$ & $0.00 \pm 0.00^{a}$ & $0.00 \pm 0.00^{\mathrm{a}}$ & $0.33 \pm 0.57^{\mathrm{a}}$ & $0.33 \pm 0.57^{a}$ & $0.33 \pm 0.57^{\mathrm{a}}$ & $0.66 \pm 0.57^{\mathrm{a}}$ & $1.00 \pm 1.00^{\mathrm{a}}$ & $6.33 \pm 3.21^{b}$ & $6.33 \pm 0.57^{b}$ \\
\hline & Aqueous & $0.00 \pm 0.00^{\mathrm{a}}$ & $0.00 \pm 0.00^{\mathrm{a}}$ & $0.00 \pm 0.00^{a}$ & $0.00 \pm 0.00^{a}$ & $0.00 \pm 0.00^{\mathrm{a}}$ & $0.00 \pm 0.00$ & $1.33 \pm 0.57^{a b}$ & $1.66 \pm 0.57^{\mathrm{ab}}$ & $2.00 \pm 1.00^{\mathrm{b}}$ & $2.33 \pm 1.52^{b}$ \\
\hline
\end{tabular}

UC: Untreated control; TC: Treated control. Values are mean of three replicates of three trials \pm standard deviation. Different superscript alphabets within the column indicate statistical significant difference in larval mortality between concentrations at $\mathrm{P}<0.05$ level by one way ANOVA followed by Tukey's test.

Table 1: Larvicidal activity of crude flower extracts of Jasminum species against Aedes aegypti at $24 \mathrm{~h}$.

\begin{tabular}{|c|c|c|c|c|c|c|c|c|c|c|c|}
\hline \multirow{2}{*}{ Plant species } & \multirow{2}{*}{ Solvents } & \multicolumn{10}{|c|}{ Concentration (mg/L) } \\
\hline & & UC & $\mathrm{TC}$ & 62.5 & 125 & 250 & 500 & 1000 & 2000 & 4000 & 8000 \\
\hline \multirow{3}{*}{$\begin{array}{l}\text { Jasminum } \\
\text { officinale }\end{array}$} & Chloroform & $0.00 \pm 0.00^{\mathrm{a}}$ & $0.00 \pm 0.00^{a}$ & $0.00 \pm 0.00^{\mathrm{a}}$ & $0.00 \pm 0.00^{\mathrm{a}}$ & $0.33 \pm 0.57^{a}$ & $0.33 \pm 0.57^{a}$ & $0.33 \pm 0.57^{a}$ & $0.33 \pm 0.57^{a}$ & $0.66 \pm 0.57^{\mathrm{ab}}$ & $2.00 \pm 1.00^{\mathrm{b}}$ \\
\hline & Methanol & $0.00 \pm 0.00^{\mathrm{a}}$ & $0.00 \pm 0.00^{a}$ & $0.33 \pm 0.57^{a}$ & $1.00 \pm 0.00^{\mathrm{a}}$ & $1.00 \pm 1.00^{\mathrm{a}}$ & $1.33 \pm 0.57^{\mathrm{a}}$ & $1.33 \pm 1.15^{\mathrm{a}}$ & $5.33 \pm 1.52^{b}$ & $19.66 \pm 0.57^{c}$ & $20.00 \pm 0.00^{c}$ \\
\hline & Aqueous & $0.00 \pm 0.00^{\mathrm{a}}$ & $0.00 \pm 0.00^{\mathrm{a}}$ & $1.00 \pm 1.00^{\mathrm{a}}$ & $1.33 \pm 1.52^{\mathrm{a}}$ & $1.66 \pm 1.15^{\mathrm{a}}$ & $1.66 \pm 0.57^{\mathrm{a}}$ & $2.00 \pm 0.00^{\mathrm{a}}$ & $2.00 \pm 1.00^{\mathrm{a}}$ & $2.00 \pm 1.00^{a}$ & $2.33 \pm 0.57^{\mathrm{a}}$ \\
\hline \multirow{3}{*}{$\begin{array}{c}\text { Jasminum } \\
\text { auriculatum }\end{array}$} & Chloroform & $0.00 \pm 0.00^{\mathrm{a}}$ & $0.00 \pm 0.00^{a}$ & $0.66 \pm 0.57^{\mathrm{ab}}$ & $1.33 \pm 0.57^{\mathrm{ab}}$ & $1.66 \pm 0.57^{a b c}$ & $1.66 \pm 0.57^{\mathrm{abc}}$ & $2.00 \pm 1.00^{\mathrm{abc}}$ & $3.00 \pm 1.00^{\mathrm{bc}}$ & $4.00 \pm 1.00^{\mathrm{cd}}$ & $6.00 \pm 2.00^{d}$ \\
\hline & Methanol & $0.00 \pm 0.00^{\mathrm{a}}$ & $0.00 \pm 0.00^{\mathrm{a}}$ & $0.00 \pm 0.00^{\mathrm{ab}}$ & $0.33 \pm 0.57^{\mathrm{abc}}$ & $0.33 \pm 0.57^{\mathrm{bcd}}$ & $0.33 \pm 0.57^{\mathrm{bcd}}$ & $0.66 \pm 0.57^{\mathrm{bcd}}$ & $2.33 \pm 0.57^{\mathrm{cd}}$ & $8.66 \pm 3.78^{\text {cd }}$ & $17.00 \pm 1.00^{d}$ \\
\hline & Aqueous & $0.00 \pm 0.00^{\mathrm{a}}$ & $0.00 \pm 0.00^{a}$ & $0.66 \pm 0.57^{\mathrm{ab}}$ & $1.66 \pm 0.57^{\mathrm{abc}}$ & $2.00 \pm 0.00^{\mathrm{bcd}}$ & $2.33 \pm 0.57^{\mathrm{bcd}}$ & $2.33 \pm 0.57^{\mathrm{bcd}}$ & $2.66 \pm 0.57^{\mathrm{cd}}$ & $2.66 \pm 1.15^{\mathrm{cd}}$ & $3.66 \pm 0.57^{d}$ \\
\hline \multirow{3}{*}{$\begin{array}{c}\text { Jasminum } \\
\text { grandiflorum }\end{array}$} & Chloroform & $0.00 \pm 0.00^{\mathrm{a}}$ & $0.00 \pm 0.00^{\mathrm{a}}$ & $7.33 \pm 0.57^{b}$ & $8.66 \pm 0.57^{\mathrm{bc}}$ & $11.33 \pm 2.30^{\mathrm{bc}}$ & $11.66 \pm 1.52^{c}$ & $20.00 \pm 0.00^{d}$ & $20.00 \pm 0.00^{d}$ & $20.00 \pm 0.00^{d}$ & $20.00 \pm 0.00^{d}$ \\
\hline & Methanol & $0.00 \pm 0.00^{\mathrm{a}}$ & $0.00 \pm 0.00^{a}$ & $1.33 \pm 0.57^{a}$ & $1.66 \pm 0.57^{a}$ & $2.66 \pm 0.57^{a}$ & $3.00 \pm 0.00^{\mathrm{ab}}$ & $3.33 \pm 1.52^{\mathrm{ab}}$ & $3.33 \pm 0.57^{\mathrm{ab}}$ & $7.66 \pm 4.61^{b}$ & $15.33 \pm 1.52^{c}$ \\
\hline & Aqueous & $0.00 \pm 0.00^{\mathrm{a}}$ & $0.00 \pm 0.00^{a}$ & $1.33 \pm 1.15^{\mathrm{ab}}$ & $1.66 \pm 0.57^{\mathrm{abc}}$ & $1.66 \pm 0.57^{a b c}$ & $1.66 \pm 0.57^{\mathrm{abc}}$ & $2.00 \pm 0.00^{\mathrm{abc}}$ & $2.66 \pm 0.57^{\mathrm{bc}}$ & $3.00 \pm 1.00^{\mathrm{bc}}$ & $3.66 \pm 1.52^{\mathrm{c}}$ \\
\hline
\end{tabular}

UC: Untreated control; TC: Treated control. Values are mean of three replicates of three trials \pm standard deviation. Different superscript alphabets within the column indicate statistical significant difference in larval mortality between concentrations at $P<0.05$ level by one way ANOVA followed by Tukey's test.

Table 2: Larvicidal activity of crude flower extracts of Jasminum species against Aedes aegypti at $48 \mathrm{~h}$. 
Citation: Eugeni Anitha Preethi G, Raveen R, Arivoli S, Samuel T, Madhanagopal R (2014) Larvicidal Efficacy of Jasminum sp. (Oleaceae) Flower Extracts against the Dengue and Chikungunya Vector Aedes aegypti L. (Diptera: Culicidae). Med chem 4: 672-675. doi:10.4172/21610444.1000210

\begin{tabular}{|c|c|c|c|c|c|c|c|c|c|c|c|}
\hline \multirow{3}{*}{ Plant species } & \multirow{3}{*}{ Solvents } & \multicolumn{10}{|c|}{ Larval mortality (\%) } \\
\hline & & \multicolumn{10}{|c|}{ Concentration (mg/L) } \\
\hline & & UC & TC & 62.5 & 125 & 250 & 500 & 1000 & 2000 & 4000 & 8000 \\
\hline \multirow{3}{*}{$\begin{array}{l}\text { Jasminum } \\
\text { officinale }\end{array}$} & Chloroform & 0.00 & 0.00 & 0.00 & 0.00 & 0.00 & 0.00 & 0.00 & 0.00 & 0.00 & 5.00 \\
\hline & Methanol & 0.00 & 0.00 & 1.65 & 1.65 & 3.30 & 3.30 & 3.30 & 6.65 & 18.30 & 18.30 \\
\hline & Aqueous & 0.00 & 0.00 & 0.00 & 0.00 & 0.00 & 0.00 & 0.00 & 0.00 & 1.65 & 3.30 \\
\hline \multirow{3}{*}{$\begin{array}{c}\text { Jasminum } \\
\text { auriculatum }\end{array}$} & Chloroform & 0.00 & 0.00 & 0.00 & 1.65 & 1.65 & 3.30 & 6.65 & 8.30 & 10.00 & 11.65 \\
\hline & Methanol & 0.00 & 0.00 & 0.00 & 0.00 & 0.00 & 0.00 & 0.00 & 5.00 & 20.00 & 20.00 \\
\hline & Aqueous & 0.00 & 0.00 & 0.00 & 3.30 & 3.00 & 6.65 & 6.65 & 8.30 & 10.00 & 10.00 \\
\hline \multirow{3}{*}{$\begin{array}{c}\text { Jasminum } \\
\text { grandiflorum }\end{array}$} & Chloroform & 0.00 & 0.00 & 33.30 & 35.00 & 36.65 & 53.30 & 100.00 & 100.00 & 100.00 & 100.00 \\
\hline & Methanol & 0.00 & 0.00 & 0.00 & 1.65 & 1.65 & 1.65 & 3.30 & 5.00 & 31.65 & 31.65 \\
\hline & Aqueous & 0.00 & 0.00 & 0.00 & 0.00 & 0.00 & 0.00 & 6.65 & 8.30 & 10.00 & 11.65 \\
\hline
\end{tabular}

Table 3: Per cent larval mortality of Aedes aegypti against crude flower extracts of Jasminum species at $24 \mathrm{~h}$.

\begin{tabular}{|c|c|c|c|c|c|c|c|c|c|c|c|}
\hline \multirow{3}{*}{ Plant species } & \multirow{3}{*}{ Solvents } & \multicolumn{10}{|c|}{ Larval mortality (\%) } \\
\hline & & \multicolumn{10}{|c|}{ Concentration (mg/L) } \\
\hline & & UC & TC & 62.5 & 125 & 250 & 500 & 1000 & 2000 & 4000 & 8000 \\
\hline \multirow{3}{*}{$\begin{array}{c}\text { Jasminum } \\
\text { officinale }\end{array}$} & Chloroform & 0.00 & 0.00 & 0.00 & 0.00 & 1.65 & 1.65 & 1.65 & 1.65 & 3.30 & 10.00 \\
\hline & Methanol & 0.00 & 0.00 & 1.65 & 5.00 & 5.00 & 6.65 & 6.65 & 26.65 & 98.30 & 100.00 \\
\hline & Aqueous & 0.00 & 0.00 & 5.00 & 6.65 & 8.30 & 8.30 & 10.00 & 10.00 & 10.00 & 11.65 \\
\hline \multirow{3}{*}{$\begin{array}{c}\text { Jasminum } \\
\text { auriculatum }\end{array}$} & Chloroform & 0.00 & 0.00 & 3.30 & 6.65 & 8.30 & 8.30 & 10.00 & 15.00 & 20.00 & 30.00 \\
\hline & Methanol & 0.00 & 0.00 & 0.00 & 1.65 & 1.65 & 1.65 & 3.30 & 11.65 & 43.30 & 85.00 \\
\hline & Aqueous & 0.00 & 0.00 & 3.30 & 8.30 & 10.00 & 11.65 & 11.65 & 13.30 & 13.30 & 18.30 \\
\hline \multirow{3}{*}{$\begin{array}{c}\text { Jasminum } \\
\text { grandiflorum }\end{array}$} & Chloroform & 0.00 & 0.00 & 36.65 & 43.30 & 56.65 & 58.30 & 100.00 & 100.00 & 100.00 & 100.00 \\
\hline & Methanol & 0.00 & 0.00 & 6.65 & 8.30 & 13.30 & 15.00 & 16.65 & 16.65 & 38.30 & 76.65 \\
\hline & Aqueous & 0.00 & 0.00 & 6.65 & 8.30 & 8.30 & 8.30 & 10.00 & 13.30 & 15.00 & 18.30 \\
\hline
\end{tabular}

Table 4: Per cent larval mortality of Aedes aegypti against crude flower extracts of Jasminum species at $48 \mathrm{~h}$.

\begin{tabular}{|c|c|c|c|c|c|}
\hline \multirow{2}{*}{ Plant species } & \multirow{2}{*}{ Solvents } & \multicolumn{2}{|c|}{$\mathrm{LC}_{50}(\mathrm{mg} / \mathrm{L})$} & \multicolumn{2}{|c|}{$\mathrm{LC}_{90}(\mathrm{mg} / \mathrm{L})$} \\
\hline & & $24 h$ & $48 h$ & $24 h$ & $48 \mathrm{~h}$ \\
\hline \multirow{3}{*}{ Jasminum officinale } & Chloroform & 16537.93 & 11439.80 & 25195.10 & 14126.81 \\
\hline & Methanol & 12652.04 & 2339.06 & 21055.59 & 3738.17 \\
\hline & Aqueous & 30135.97 & 17013.18 & 55517.87 & 23589.38 \\
\hline \multirow{3}{*}{ Jasminum auriculatum } & Chloroform & 17045.78 & 11001.98 & 28289.38 & 20180.45 \\
\hline & Methanol & 10593.40 & 5044.02 & 16353.63 & 8019.21 \\
\hline & Aqueous & 22427.85 & 18829.36 & 38694.80 & 35563.24 \\
\hline \multirow{3}{*}{ Jasminum grandiflorum } & Chloroform & 344.01 & 300.47 & 762.68 & 761.07 \\
\hline & Methanol & 8880.63 & 5203.02 & 14467.53 & 9963.65 \\
\hline & Aqueous & 17839.42 & 15031.87 & 33425.77 & 24108.36 \\
\hline
\end{tabular}

Table 5: Probit analysis of crude flower extracts of Jasminum species against Aedes aegypti.

The results of the present study corroborate with earlier reports of plant extracts tested against the larvae of Aedes aegypti viz., the dichloromethane extract of aerial parts of Pterocaulon polystachium $\left(\mathrm{LC}_{50} 149.2 \mathrm{ppm}\right)$ [23], ethyl acetate leaf extract of Sphaeranthus indicus $\left(\mathrm{LC}_{50} 201.11 \mathrm{ppm}\right)$ [24], hexane leaf extract of Abutilon indicum $\left(\mathrm{LC}_{50}\right.$ $261.31 \mathrm{ppm})$ [25], ethyl acetate leaf extract of Leucas aspera $\left(\mathrm{LC}_{50} 483.21\right.$ ppm) [26]. Kamaraj et al. [27] have reported larvicidal efficacy of Cassia auriculata flower methanol extracts against the larvae of Anopheles subpictus and Culex tritaeniorhynchus. Mathew et al. [28] reported the chloroform extract of Nyctanthes arbortristis leaves to possess larvicidal activity against the Aedes aegypti with $\mathrm{LC}_{50}$ value of $526.3 \mathrm{ppm}$ and its flower methanol extracts with $679.4 \mathrm{ppm}$. The chloroform extract of Orthosiphon thymiflorus exhibited larvicidal activity with $\mathrm{LC}_{50}$ value of 197.91 ppm against Aedes aegypti [29]. The chloroform leaf extract of Acalypha alnifolia when tested against Aedes aegypti showed larvicidal activity with $\mathrm{LC}_{50}$ value of $182.58 \mathrm{ppm}$ [30].

The preliminary screening of plant extracts against mosquitoes is a good means of evaluating the potential mosquitocidal property present in it $[13,31]$. Natural insecticides of plant origin have been given importance due to their ecofriendly nature and biodegradability as a substitute of synthetic insecticides for the control of vectors of public health importance. Plants are the chemical factories and rich source of bioactive chemicals, some of which have medicinal and pesticidal properties [32]. Different types of phytochemicals of plant either from the whole part or from the specific parts come out with solvent during chemical extraction depending on the polarity of the solvent [33-35]. The botanical extracts from the plant leaves, roots, seeds, flowers and bark in their crude form have been used as conventional insecticides for centuries. The complex mixtures of phytocompounds can be used to develop environmentally-safe vector and pest-managing agents. In conclusion, the results reported in the present study open the possibility for further investigations of the efficacy of larvicidal properties of the crude chloroform extracts of Jasminum grandifloruma against Aedes aegypti as a potential agent for combating mosquitoes. Further investigations are needed to elucidate this activity against a wide range of all stages of mosquito species and also to identify the active ingredient(s) of the extract responsible for larvicidal activity.

\section{References}

1. WHO (2010) Malaria fact sheets No. 94, WHO Report, Geneva, WHO media centre. 
Citation: Eugeni Anitha Preethi G, Raveen R, Arivoli S, Samuel T, Madhanagopal R (2014) Larvicidal Efficacy of Jasminum sp. (Oleaceae) Flower Extracts against the Dengue and Chikungunya Vector Aedes aegypti L. (Diptera: Culicidae). Med chem 4: 672-675. doi:10.4172/21610444.1000210

2. VCRC (1989) Vector control research centre (Ed: Rajagopalan, P.K.). Miscellaneous Publications 11: 26.

3. Selvaraj M, Mosses M (2011) Efficacy of Melia azedarach on the larvae of three mosquito species Anopheles stephensi, Culex quinquefasciatus and Aedes aegypti (Diptera: Culicidae). Eur Mosq Bull 29: 116-121.

4. Cetin H, Erler F, Yanikoglu A (2004) Larvicidal activity of a botanical natural product, AkseBio2, against Culex pipiens. Fitoterapia 75: 724-728.

5. Mandal S (2012) Mosquito vector management with botanicals - the most effective weapons in controlling mosquito-borne diseases. Asian Pac J Trop Biomed 2: 336

6. Shaalan EA, Canyon D, Younes MW, Abdel-Wahab H, Mansour AH (2005) A review of botanical phytochemicals with mosquitocidal potential. Environ Int 31: 1149-1166

7. Sakthivadivel M, Daniel T (2008) Evaluation of certain insecticidal plants for the control of vector mosquitoes, viz., Culex quinquefasciatus, Anopheles stephensi and Aedes aegypti. Appl Entomol Zool 43: 57-63.

8. Arivoli S, Ravindran KJ, Samuel T (2012) Larvicidal efficacy of plant extracts against the malarial vector Anopheles stephensi Liston (Diptera: Culicidae). World J Med Sci 7: 77-80.

9. Ghosh A, Chowdhury N, Chandra G (2012) Plant extracts as potential mosquito larvicides. Ind J Med Res 135: 581-598.

10. Raveen R, Dhayanidhi P, Dhinamala K, Arivoli S, Samuel T (2012) Larvicidal activity of Pedilanthus tithymaloides (L.) Poit (Euphorbiaceae) leaf against the dengue vector Aedes aegypti (L.) (Diptera: Culicidae). International Journal of Environmental Biology 2: 36-40.

11. Raveen R, Kamakshi KT, Deepa M, Arivoli S, Samuel T (2014) Larvicidal activity of Nerium oleander L. (Apocynaceae) flower extracts against Culex quinquefasciatus Say (Diptera: Culicidae). Int J Mosq Res 1: 36-40.

12. Samuel T, Ravindran KJ, Arivoli S (2012) Bioefficacy of botanical insecticides against the dengue and chikungunya vector Aedes aegypti (L.) (Diptera: Culicidae). Asian Pac J Trop Biomed 2: S1842-S1844.

13. Samuel T, Ravindran KJ, Arivoli S (2012) Screening of twenty five plant extracts for larvicidal activity against Culex quinquefasciatus Say (Diptera: Culicidae). Asian Pac J Trop Biomed 2: S1130-S1134

14. WHO (2005) Guidelines for laboratory and field testing of mosquito larvicides Geneva.

15. Abbott WS (1925) A method of computing the effectiveness of an insecticide. $J$ Econ Entomol 18: 265-267.

16. Unsal A, Tozun M, Parspour A, Gorenek B, Tirpan K (2007) Distribution of blood pressure among men in EskiÅŸehir. Anadolu Kardiyol Derg 7 Suppl 1 : 201-205.

17. Chandre F, Darriet F, Darder M, Cuany A, Doannio JM, et al. (1998) Pyrethroid resistance in Culex quinquefasciatus from west Africa. Med Vet Entomol 12: 359-366.

18. Liu H, Xu Q, Zhang L, Liu N (2005) Chlorpyrifos resistance in mosquito Culex quinquefasciatus. J Med Entomol 42: 815-820.

19. Kalyanasundaram M, Das PK (1985) Larvicidal \& synergestic activity of plant extracts for mosquito control. Ind J Med Res 82: 19-23.

20. Govindarajan M, Jebanesan A, Pushpanathan T (2008) Larvicidal and ovicida activity of Cassia fistula Linn leaf extract against filarial and malarial vector mosquitoes. Parasitol Res 102: 289-292.

21. Prabhu K, Murugan K, Nareshkumar A, Ramasubramanian N, Bragadeeswaran $S$ (2011) Larvicidal and repellent potential of Moringa oleifera against malaria vector, Anopheles stephensi Liston (Insecta: Diptera: Culicidae). Asian Pac J Trop Biomed 1: 127-132.

22. Rahuman AA, Venkatesan $P$ (2008) Larvicidal efficacy of five cucurbitaceous plant leaf extracts against mosquito species. Parasitol Res 103: 133-139.

23. Ciccia G, Coussio J, Mongelli E (2000) Insecticidal activity against Aedes aegypti larvae of some medicinal South American plants. J Ethnopharmaco 72: $185-189$.
24. Samuel T, Arivoli S (2011) Larvicidal, adult emergence inhibition and ovicidal activity of Sphaeranthus indicus Linn. (Asteraceae) whole plant extracts against Aedes aegypti, Anopheles stephensi and Culex quinquefasciatus (Diptera: Culicidae). Ind J Environ Ecoplann 18: 293-304.

25. Arivoli S, Samuel T (2011a) Larvicidal and adult emergence inhibition activity of Abutilon indicum (Linn.) (Malvaceae) leaf extracts against vector mosquitoes (Diptera: Culicidae). J Biopest 4: 27-35

26. Arivoli S, Samuel T (2011b) Effects of Leucas aspera (Willd.) Spreng (Lamiaceae) leaf extracts against Aedes aegypti, Anopheles stephensi and Culex quinquefasciatus (Diptera: Culicidae). World Appl Sci J 14: 565-568.

27. Kamaraj C, Bagavan A, Rahuman AA, Zahir AA, Elango G, et al. (2009) Larvicidal potential of medicinal plant extracts against Anopheles subpictus Grassi and Culex tritaeniorhynchus Giles (Diptera: Culicidae). Parasitol Res 104: 1163-1171.

28. Mathew N, Anitha MG, Bala TS, Sivakumar SM, Narmadha R, et al. (2009) Larvicidal activity of Saraca indica, Nyctanthes arbortristis and Clitoria ternatea extracts against three mosquito vector species. Parasitol Res 104: 1017-1025.

29. Kovendan, K., Murugan, K., Vincent, S. and Barnard, D.R. 2012a. Mosquito larvicidal properties of Orthosiphon thymiflorus (Roth) Sleensen. (Labiatae) against mosquito vectors, Anopheles stephensi, Culex quinquefasciatus and Aedes aegypti (Diptera: Culicidae). Asian Pac J Trop Med 2: 412-420.

30. Kovendan K, Murugan K, Vincent S (2012b) Evaluation of larvicidal activity of Acalypha alnifolia Klein ex Willd. (Euphorbiaceae) leaf extract against the malarial vector, Anopheles stephensi, dengue vector, Aedes aegypti and bancroftian filariasis vector, Culex quinquefasciatus (Diptera: Culicidae) Parasitol Res 110: 571-581.

31. Kumar S, Nair G, Singh AP, Batra S, Wahab N, et al. (2012) Evaluation of stem roots and leaves of the weed, Parthenium hysterophorus (Family: Asteraceae) against Aedes aegypti L. Asian Pacific Journal of Tropical Disease 2: 395-400.

32. Chansang U, Zahiri NS, Bansiddhi J, Boonruad T, Thongsrirak $P$, et al (2005) Mosquito larvicidal activity of aqueous extracts of long pepper (Piper retrofractum vahl) from Thailand. J Vector Ecol 30: 195-200.

33. Sukumar K, Perich MJ, Boobar LR (1991) Botanical derivatives in mosquito control: a review. J Am Mosq Cont Assoc 7: 210-237.

34. Chowdhury N, Bhattacharjee I, Laskar S, Chandra G (2007) Efficacy of Solanum villosum Mill. (Solanaceae: Solanales) as biocontrol agent against fourth instar larvae of Culex quinquefasciatus Say. Turk J Zool 31: 365-370.

35. Rawani A, Ghosh A, Laskar S, Chandra G (2012) Aliphatic amide from seeds of Carica papaya as mosquito larvicide, pupicide, adulticide, repellent and smoke toxicant. J Mosq Res 2: 8-14 